\title{
CLIMA FAMILIAR Y AGRESIVIDAD EN ESTUDIANTES DE SECUNDARIA DE LIMA METROPOLITANA
}

\author{
FAMILY CLIMATE AND AGGRESSIVENESS BETWEEN HIGH SCHOOL STUDENTS \\ FROM METROPOLITAN LIMA
}

Maria Matalinares C. ${ }^{1}$; Carlos Arenas I.; Lidia Sotelo L.; Gloria Diaz A.; Alejandro Dioses Ch.; juan Yaringaño L.; Raúl Muratta E.; Cecilia Pareja F.; Rina Tipacti T.

Universidad Nacional Mayor de San Marcos, Perú

(RECiBIDo el 26/02/2010, ACEPTADo El 15/06/2010)

\begin{abstract}
RESUMEN
La investigación realizada tuvo como objetivo establecer si existía o no relación entre el clima familiar y la agresividad de los estudiantes de cuarto y quinto de secundaria de Lima Metropolitana. Para la realización del proyecto se evaluó a 237 estudiantes de cuarto y quinto de secundaria, de ambos sexos, cuyas edades fluctuaron entre los 14 y 18 años de edad, procedentes de diversos centros educativos estatales de Lima, a quienes se aplicó el Inventario de hostilidad de Buss-Durkee, propuesto por A. H. Buss en 1957 y adaptado a nuestro país por Carlos Reyes R. en 1987, y la Escala del clima social en la familia (FES), de los autores: R. H. Moos. y E. J. Trickett, cuya estandarización fue realizada por Cesar Ruiz Alva y Eva Guerra Turín (1993). Al ser procesados los resultados se encontró que las variables clima familiar y agresividad se encuentran correlacionadas. Al analizar los resultados tomando en cuenta los diversos subtests de la Escala de clima social se encontró que la dimensión Relación de la escala de clima social se relaciona con las subescalas hostilidad y agresividad verbal. No se encontró una relación significativa entre la dimensión desarrollo del clima social en la familia y las subescalas del cuestionario de agresividad. El clima social familiar de los estudiantes se muestra diferente en función del sexo al hallarse diferencias significativas en la dimensión estabilidad entre varones y mujeres. La agresividad de los estudiantes también se muestra diferente en función del sexo, al hallarse diferencias significativas en la subescala de agresividad física entre varones y mujeres.
\end{abstract}

Palabras claves: Estudiantes; clima social en la familia; agresividad.

\begin{abstract}
The present research work started with an objective of establish if it existed or not relation between family climate and aggressiveness in students of 4th and 5th of high school from Metropolitan Lima. The sample comprised 237 students of 4th and 5th of high school level, of both sexes, with aged between 14 and 18 years, from different educational state centers of Lima to whom were applied the Hostility Inventory of Buss-Durkee, proposed for A. H. Buss and A. Durkee in 1957, adapted for Peru by Carlos Reyes R. in 1987 and

1 Docente principal de la Facultad de Psicología de la UNMSM.

E-mail: maria_luisa93@hotmail.com.
\end{abstract}


the Family Environment Scale (FES) by R.H. Moos, B.S. Moos and E.J. Trickett whose standardization was realized by Cesar Ruiz. The results indicate that the variables family climate and aggressiveness are correlated. Besides, the results about different subtests of the Family Environment Scale show a correlation between the dimension Relation of Family Environment Scale with the subscales Hostility and Verbal Aggressiveness. There was not a relation between the dimension Development of the Family Environment Scale and the subscales of the Hostility Inventory. There was significant differences on genre in high school students about Family Environment Scale, particularly in the dimension Stability between males and females. The aggressiveness of the students also show significant differences on the genre in the subscale of physical aggressiveness between males and females.

Keywords: Students; family social climate; aggressiveness.

\section{INTRODUCCIÓN}

Benites (1997) afirma que la familia sigue siendo considerada como la estructura básica de la sociedad, cuyas funciones no han podido ser sustituidas por otras organizaciones creadas expresamente para asumir sus funciones. De estas, la más importante es aquella de servir como agente socializador que permita proveer condiciones y experiencias vitales que facilitan el óptimo desarrollo bio-psico-social de los hijos. Por su parte, Zavala (2001) define a la familia como el conjunto de personas que viven juntas, relacionadas unas con otras, que comparten sentimientos, responsabilidades, informaciones, costumbres, valores, mitos y creencias. Cada miembro asume roles que permiten el mantenimiento del equilibrio familiar. Es una unidad activa, flexible y creadora; una institución que resiste y actúa cuando lo considera necesario. Red de relaciones vividas. La familia como institución social es un sistema de fuerzas que constituyen un núcleo de apoyo para sus miembros y la comunidad.

El clima social es un concepto que pretende describir las características psicosociales e institucionales de un determinado grupo asentado sobre un ambiente. Zavala (2001) define el clima familiar como el estado de bienestar resultante de las relaciones que se dan entre los miembros de la misma. Dicho estado refleja el grado de comunicación, cohesión e interacción, siendo esta conflictiva o no así como el nivel de organización con que cuenta la familia y el control que ejercen unos sobre otros.

En cuanto al clima social familiar, son tres las dimensiones o atributos afectivos que hay que tener en cuenta para evaluarlo: una dimensión de relaciones, una dimensión de desarrollo personal, y una dimensión de estabilidad y cambio de sistemas; las cuales se dividen a su vez en subescalas. Para estudiar estas dimensiones, Moos (1974) ha elaborado diversas escalas de clima social aplicables a diferentes tipos de ambiente como es el caso de la escala de clima social en la familia (FES). En el clima familiar se dan interrelaciones entre los miembros de la familia donde se dan aspectos de comunicación, interacción, etc. El desarrollo personal puede ser fomentado por la vida en común, así como la organización y el grado de control que se ejercen unos miembros sobre otros.

En el clima social familiar la dimensión denominada "relaciones" evalúa el grado de comunicación y libre expresión dentro de la familia y el grado de interacción conflictiva que la caracteriza. Está formada por las subescalas de "cohesión” (CO), definida como 
el grado en que los miembros de la familia se apoyan y ayudan entre sí; la subescala de "expresividad" (EX), definida como el grado en que se permite a los miembros de la familia expresar libremente sus sentimientos, y la de "conflicto" (CT), descrita como el grado en que se expresan abiertamente la cólera, la agresividad y el conflicto entre los miembros de la familia. La dimensión definida como "desarrollo" evalúa la importancia que tienen dentro de la familia, ciertos procesos de desarrollo personal que pueden ser fomentados o no por la vida en común. Está constituida por cinco subescalas: "autonomía" (AU) o grado en que los miembros de la familia están seguros de sí mismos y toman sus propias decisiones; "actuación" (AC), referida al grado en que las actividades se enmarcan en una estructura competitiva; "intelectual cultural" (IC) o grado de interés en las actividades políticas, sociales, intelectuales y culturales; "social recreativo" (SR) que mide el grado de participación en este tipo de actividades, y la "moralidad-religiosidad" (MR) definida por la importancia que se da en el ámbito familiar a las prácticas y valores de tipo ético y religioso. Finalmente, la dimensión de "estabilidad" da información sobre la estructura y organización de la familia y sobre el grado de control que unos miembros de la familia ejercen sobre otros. Está formada por dos subescalas: "organización” (ORG) que evalúa la importancia que se da a la organización y estructura al planificar las actividades y responsabilidades de la familia, y la subescala de "control" (CTL) o grado en que la dirección de la vida familiar se atiene a reglas y procedimientos establecidos (Ruiz y Guerra, 1993).

Se han realizado investigaciones en el extranjero con el clima familiar. En España, Jiménez, M.; Ferró, M.; Gómez, R. y Parra, P. (1999) evaluaron el clima familiar en una muestra de 923 estudiantes de secundaria de 13 a 16 años, con la escala de FES de Moos. Entre otros, los resultados mostraron que existe relación entre el conflicto en los ambientes familiares con características de baja cohesión y bajo nivel de organización familiar. Se evidenció también que los adolescentes pertenecientes a familias con alto nivel de control manifiestan menor expresividad y autonomía, y los que pertenecen a familias con alto nivel en actividad social, recreativa y en intereses culturales afirman relacionarse con mayores números de amigos.

Rodríguez y Torrente (2003) investigaron la interacción familiar y la conducta antisocial en Murcia con 641 estudiantes de ESO y bachillerato, cuyas edades fluctuaban entre 11 y 17 años, a quienes aplicaron la escala de clima social familiar de Moos, un cuestionario sobre estilos educativos de los padres y un cuestionario de conductas antisociales. Los resultados mostraron que los chicos se involucran en más actividades delictivas que las chicas. Las familias de los menores que pertenecen al grupo adaptados obtienen puntuaciones medias más elevadas en las subescalas de cohesión, expresividad, moralidad-religiosidad y organización, y más bajas en las escalas de conflicto y social-recreativo.

En México, Vera, Morales y Vera (2005) investigaron la relación del desarrollo cognitivo con el clima familiar y el estrés de la crianza. En una muestra de 120 familias en pobreza extrema de las zonas marginales de Hermosillo, Sonora, México. Madres de 32 años en promedio respondieron a una escala de funcionamiento familiar, estrés de la crianza, y sus niños fueron medidos en desarrollo cognitivo. El objetivo fue evaluar las diferencias significativas entre los tres tipos de familias en su desarrollo cognitivo, el estrés de la 
crianza y el clima familiar. Se clasificaron a las familias en base a la medida de clima familiar con un análisis de conglomerados (clusters). Los resultados indican: a) tres tipos de climas que etiquetamos como: disciplinado, cohesionado y sin orientación; b) el clima sin orientación se asocia a una percepción negativa del niño y de restricciones por la maternidad, y presentan las medias más bajas de desarrollo cognitivo, y c) el clima cohesivo se asocia con menos estrés en la madre por la crianza y los puntajes más alto de desarrollo cognitivo.

En Venezuela, Montiel-Nava, Montiel-Barbero y Peña (2005) investigaron el efecto del clima familiar en el desarrollo del trastorno por déficit de atención-hiperactividad. Recolectaron datos demográficos, escalas de puntuación de padres y profesores, cociente intelectual y la escala de clima familiar (ECF), de 53 niños (edades 4 a 13 años); 29 con diagnóstico de TDAH y 24 no casos. Los resultados mostraron diferencias significativas entre casos y controles para las subescalas de cohesión, orientación hacia actividades intelectuales y orientación hacia actividades recreativas. Una mayor gravedad en síntomas de TDAH estuvo relacionada con menor cohesión y mayor conflicto familiar. Los hallazgos sugieren falta de asociación entre la calidad del clima familiar y el diagnóstico del TDAH.

En el 2006, un grupo de investigadores de la universidades UPV, UNED y UBA: Darío Páez, Myriam Campos, Itziar Fernández, Elena Zubieta y María Martina Casullo investigaron el apego seguro, vínculos parentales, clima familiar e inteligencia emocional: socialización, regulación y bienestar. Los resultados mostraron que un estilo de socialización emocional infantil, centrado en la expresividad y calidez como un clima familiar expresivo, cohesivo, bajo en conflicto y promotor de la independencia, son correlatos importantes de la inteligencia emocional y de una mayor felicidad en los jóvenes.

Moreno, Vacas y Roa (2006) en Granada, España investigaron la violencia escolar con diez situaciones del ambiente sociofamiliar de los individuos de una muestra de 1119 escolares comprendidos entre 8 y 17 años, y su relación con el clima social familiar. Los resultados indicaron la fuerte relación entre aspectos del ámbito sociofamiliar como el control familiar, la cohesión, el conflicto, la expresividad o los intereses culturales y sociorecreativos, con factores de violencia escolar (victimizado, victimizador, y trato en colegio y casa). También se han encontrado diferencias significativas en la edad, en la que los individuos menores de 12 años se manifestaron más afectados por la violencia escolar, y el sexo, en el que los varones indicaron estar más involucrados en fenómenos de bullying, la relación entre la victimización y el clima sociofamiliar.

Ahora bien, en el Perú se han realizado algunas investigaciones sobre clima familiar. Guerra (1993) estudió las características del clima social familiar y su relación con el rendimiento académico de una muestra de 180 alumnos de ambos sexos pertenecientes a un colegio estatal.

Salinas, Rojas, Matos y Zegarra (2005) evaluaron 126 familias de alumnos de cuarto y quinto de secundaria. La muestra fue evaluada con el Inventario de autoestima y la escala social familiar de Moos. Los resultados mostraron que existía un moderado grado de la valoración de la autoestima, la mayoría de las familias dan importancia a la organización y 
planificación de sus actividades, además hallaron que existía un escaso grado de dirección familiar en función a reglas y procedimientos establecidos.

Escrivá, García y Pérez (2001) investigaron la importancia del entorno familiar en el desarrollo del concepto personal. La muestra estuvo formada por 333 participantes varones y mujeres, con una media de edad de 15.08 años en la primera evaluación y de 16.38 en el retest. Los resultados indican que las variables personales, edad y sexo modulan el autoconcepto de los adolescentes. En relación al clima familiar, los factores de cohesión, expresividad y organización familiar guardan relación positiva con todas las áreas del autoconcepto, mientras que la conflictividad familiar es el factor que mantiene la relación negativa.

Zavala (2001) investigó la relación entre las dimensiones del clima familiar, los intereses vocacionales y los tipos caracterológicos de los alumnos del quinto año de secundaria de los colegios nacionales del distrito del Rímac. Los resultados revelaron que el $39.2 \%$ de los evaluados vivencian inadecuados niveles de comunicación, interacción y libre expresión al interior del seno familiar, en contraposición a un $6.9 \%$ que presenta buenas relaciones. El $47.6 \%$ de la muestra comunica que no se sienten apoyados por los miembros de su familia al momento de tomar decisiones o de actuar, además de no encontrar oportunidades para el desarrollo de sus potencialidades, a diferencia de un $15.8 \%$ que refiere tener buenas condiciones para desarrollarse. El 53.5\% de los evaluados expresan que el clima familiar que vivencian presenta una estructura inadecuada, caracterizada por la inestabilidad, mientras que ningún alumno refiere vivenciar un ambiente estable en su hogar.

García (2005) investigó la relación entre habilidades sociales, clima social familiar y rendimiento académico en estudiantes universitarios. Para su realización se evaluó a 205 estudiantes, de ambos sexos, mayores de 16 años, que cursaban el primer ciclo de estudios en psicología, procedentes de la Universidad Particular de San Martín de Porres y de la Universidad Nacional Mayor de San Marcos, a quienes se aplicó la Lista de chequeo de habilidades sociales de Goldstein, traducida y adaptada a nuestro país por Ambrosio Tomás entre 1994-1995, y la escala de clima social en la familia de Moos y Trickett estandarizada en el Perú por Ruiz y Guerra en 1993. Los resultados de la investigación fueron procesados por el coeficiente de correlación de Pearson, prueba Chi cuadrado, media y desviación estándar, el coeficiente alfa de Cronbach. Los resultados arrojaron una correlación positiva y significativa entre habilidades sociales y el clima social en la familia, encontrándose también que no existe estadísticamente una correlación significativa entre habilidades sociales y clima social en la familia con el rendimiento académico; se encontró además que en relación con las variables habilidades sociales y clima social en la familia, más del $50 \%$ de los alumnos de la muestra total se ubicaron en el nivel promedio (65.9\% y $62.7 \%$ respectivamente) y en rendimiento académico solo un porcentaje bajo de alumnos $(9.8 \%)$ obtuvo notas desaprobatorias.

Respecto de la variable agresividad, Berkowitz (1996) la define como un "estado emocional que consiste en sentimientos de odio y deseos de dañar a otra persona, animal u objeto". Hurlock (2000) se refiere a la agresividad como un acto real o amenazado de hostilidad, provocado por otra persona. Se manifiesta mediante ataques físicos o verbales hacia otro que generalmente es menor que el agresor; es decir, que una persona en mejores condiciones que otra trata de imponer sus reglas para lograr su propósito. 
Para Buss (1992), las respuestas agresivas poseen dos características: la descarga de estímulos nocivos y un contexto interpersonal; definiendo a la agresión como una reacción que descarga estímulos nocivos sobre otros organismos.

La definición de los factores permite desglosar los niveles conductual, emocional y cognitivo de la agresividad, lo cual facilita el estudio dentro del enfoque cognitivo-conductual. Los ítemes se distribuyen en cuatro factores bien definidos: agresión física, agresión verbal, ira y hostilidad. Los dos primeros explican la dimensión conductual; el segundo, la emocional, y el último se refiere al factor cognitivo de la agresividad Buss y Perry (1992).

Para Wesinger (1988), la ira es una reacción cognitiva emocional fisiológica ante afrentas percibidas por el individuo, intrusiones en el terrero personal y/o frustraciones de la conducta dirigidas hacia un fin, en los que los procesos cognitivos a menudo distorsionan la realidad y generan más ira.

Lazarus y Lazarus (2000) señalan que la hostilidad generalmente se refiere a un sentimiento y no a una emoción; es decir, las personas se sienten hostiles hacia otras personas cuando hay una predisposición a enojarse con alguien, tanto si una acción ofensiva es provocadora, como si no lo es pero es interpretada como provocadora. La persona siempre siente esa hostilidad específica hacia alguien o hacia algo, pero solo se enoja o siente ira cuando él decide sentirla. En la perspectiva de estos autores, el enojo está asimilado con la ira, la cual puede aparecer como resultado de una ofensa humillante contra la persona o contra sus seres queridos. Las situaciones que lo producen pueden ser diversas; en ellas pueden existir ataques fuertes y directos (un comentario ofensivo, un insulto entre otros) o ataques sutiles, moderados o ambiguos (provocaciones sutiles ambiguas). La agresividad es diferente a la ira, teniendo en cuenta que cuando se está irascible o enojado hay un impulso de atacar con mayor fuerza y a veces es difícil de controlar, lo que ya sería agresividad.

Cuando se habla del comportamiento agresivo se hace referencia a una multicausalidad, pues tal comportamiento está influenciado por diferentes factores: social, cultural, genético y biológico, tanto a nivel familiar como individual, justificándose así el abordaje desde diversos enfoques teóricos (Castrillón, D.; Ortiz, A. y Vieco, F., 2004, citados por Castillo, 2006).

Ahora bien, es importante la influencia de los padres y del entorno familiar sobre el modo de manejar la agresividad del niño. Al respecto Gaspar (1999, p. 23) explica que es determinante para manejar la agresividad del niño la forma cómo los padres ejercen su autoridad, firmeza y disciplina. Y también el hecho de que muestren permisividad, falta de límites o aceptación pasiva de las conductas inadecuadas del niño. Por ello agrega que la socialización de la agresividad tiene que ocurrir en el ámbito familiar, si hay fallas en ese entorno o el ambiente familiar natural no existe o es sustituido por otro, la situación en el futuro del niño cambia radicalmente.

Castrillon y Vieco (2002) realizaron una investigación en la ciudad de Medellín con 500 sujetos, 258 mujeres y 242 hombres acerca de las actitudes mediante las cuales los estudiantes de dos universidades públicas (Universidad de Antioquia y Universidad Nacional Seccional Medellín) y una privada (Universidad Cooperativa de Colombia) justifican sus comportamientos agresivos y violentos. Se analiza cómo la población estudiada acude a 
diferentes tipos de agresión como la directa, la indirecta, la física o la verbal en situaciones en que las consideran justificables. Los resultados mostraron que los hombres acuden más a la agresión física y directa en las universidades estudiadas, mientras que las mujeres utilizan tipos de agresión verbal e indirecta.

En Granada, España, Vacas (2002) investigó la relación entre la agresividad escolares y las dificultades de aprendizaje en una muestra de 352 alumnos de cuarto primaria hasta el segundo de bachillerato, cuyas edades fluctuaban entre los 9 y 16 años, de nivel socioeconómico medio. Los resultados mostraron que hay una tendencia de los sujetos con dificultades en el aprendizaje a asumir con mayor frecuencia los roles de víctima y en menor medida el rol de matón.

Noroño, Cruz, Cadalso y Fernández (2002) realizaron un estudio descriptivo sobre la influencia del medio familiar en niños de 9 a 11 años, con conductas agresivas, evaluados y diagnosticados en el departamento de psicología del policlínico “ Dr. Tomás Romay”; la muestra estuvo conformada, además, por los padres de estos niños. A todos ellos se les aplicó una serie de técnicas con el objetivo principal de describir las características del medio familiar y su influencia en las conductas agresivas de los infantes. Se concluyó que predominaron las familias disfuncionales incompletas, con manifestaciones de agresividad, alcoholismo, mala integración social y familiar, rechazo hacia los hijos e irresponsabilidad en su cuidado y atención.

Muñoz, Carreras y Braza (2004), en Cádiz, España, analizaron cómo los componentes de la competencia social se relacionan con la presencia de comportamientos disruptivos en una muestra de 348 alumnos de un centro de secundaria. En el primer ciclo de ESO, los chicos disruptivos obtuvieron puntuaciones más altas en liderazgo prosocial, en agresividad y terquedad, y en impulsividad; las chicas de este ciclo obtuvieron puntuaciones más bajas en sensibilidad social. En el segundo ciclo de ESO, entre los chicos no se detectaron diferencias estadísticamente significativas entre los sujetos disruptivos y los no disruptivos; entre las chicas, las calificadas como disruptivas obtuvieron puntuaciones más bajas en ayuda y colaboración y conformidad con lo que es socialmente correcto, y más altas en independencia frente a dependencia de campo.

Ortega y Monk (2005) analizaron la naturaleza de la agresión injustificada en una muestra de preescolares de la ciudad de Sevilla. Los resultados mostraron que los preescolares tienden a usar la agresión directa (cara a cara) más que la agresión indirecta; los agresores no eran percibidos como tales pero eran más rechazados que los demás; los defensores resultaron ser los más populares; las victimas no aparecieron ni como más débiles, ni como más rechazadas.

Kaleel, Justicia, Benítez y Pichardo (2007) realizaron una investigación en Palestina con una muestra de 1492 alumnos de educación primaria y secundaria cuyas edades fluctuaban entre los 10 y 16 años en centros educativos de la zona urbana y rural de Hebrón. Los resultados mostraron que el $53.5 \%$ de los sujetos que informan haber agredido a alguno de sus compañeros, al menos una vez por semana, son hombres, frente al $46.5 \%$ de las mujeres. Al igual que en el caso anterior, las diferencias entre hombres y mujeres resultan estadísticamente significativas. El porcentaje de hombres agresores aumenta en función de la frecuencia. Así, los sujetos varones que agreden a sus compañeros dos o tres veces por 
semana representan el $88 \%$ de los agresores. Los resultados encontraron también tasas de incidencia del maltrato similares a las encontradas en los países occidentales: $11.1 \%$ de víctimas, $9.5 \%$ de agresores y $78.2 \%$ de espectadores. Sin embargo, los datos también ponen de manifiesto que un mayor número de alumnos palestinos, en comparación con estudios occidentales, están involucrados en episodios de maltrato hacia los compañeros, ya sea como víctimas o como agresores. De la misma forma, los datos confirman tendencias similares en el fenómeno de los malos tratos entre iguales, entre el alumnado palestino y las encontradas en el alumnado occidental.

Murcia, Reyes, Gómez, Medina, Paz y Fonseca (2007) en su estudio realizado en Honduras con una muestra de 576 escolares de ambos sexos, cuyas edades fluctuaban entre los 10 y 15 años, procedentes de escuelas públicas y privadas a quienes se aplicó el Inventario de hostilidad de Buss y Burke, y una entrevista familiar semiestructurada. Los resultados mostraron que los niños con mayores niveles de hostilidad y agresividad proceden de hogares desintegrados y disfuncionales, con evidencias de violencia doméstica y alcoholismo.

En el Perú, Díaz (1987) realizó un estudio retrospectivo, transversal y descriptivo en el Instituto nacional de salud mental "Honorio Delgado-Hideyo Noguchi" a fin de identificar la influencia del ambiente familiar y de las actitudes parentales en las conductas agresivas que presenta el preescolar, así como establecer la relación entre tipos de agresividad con los factores predisponentes como: estado civil, métodos disciplinarios y grado de instrucción de los padres. La población objeto de estudio estuvo constituida por 180 niños de ambos sexos de 3 a 6 años de edad, que presentaron conductas agresivas cuyas historias clínicas fueron revisadas sistemáticamente. Los resultados mostraron que existe relación estadística significativa entre las actitudes parentales y la conducta agresiva de los preescolares; mas no existe asociación estadística con la variable sexo; así mismo se observó que existe mayor proporción de niños agresivos en relación a las niñas y que la agresividad se incrementa con la edad. Los resultados del estudio revelan también que la forma de conducirse de los padres, su trato para con sus hijos y la forma de disciplinar y educar a estos influyen en las manifestaciones agresivas que presentan.

Hernández (1988) realizó un estudio sobre la relación entre las actitudes parentales y la conducta agresiva de los preescolares. Estudió a 180 niños de ambos sexos de 3 a 6 años de edad que presentaron conductas agresivas cuyas historias clínicas fueron revisadas sistemáticamente. Los resultados mostraron que existe relación estadística significativa entre las actitudes parentales y la conducta agresiva de los preescolares; mas no existe asociación estadística con la variable sexo; así mismo se observó que existe mayor proporción de niños agresivos en relación a las niñas y que la agresividad se incrementa con la edad. Los resultados del estudio revelan también que la forma de conducirse de los padres, su trato para con sus hijos y la forma de disciplinar y educar a estos influye en las manifestaciones agresivas que presentan.

Rodríguez y Tincopa (2006) investigaron la relación entre el soporte social y agresividad en adolescentes infractores del centro juvenil "Trujillo". En una muestra de 62 internos del centro juvenil Trujillo, cuyas edades fluctuaban entre los 14 y 18 años, aplicaron el Inventario de hostilidad de Buss y Durkee, y la escala de soporte social de Falherthy, 
Gaviria y Pathak. Los resultados mostraron que no existe una correlación sustancial entre el soporte social y la agresividad en los adolescentes infractores del centro juvenil Trujillo, pero hallaron correlaciones entre la subescala de resentimiento y el área de apoyo emocional, correlación entre la subescala de agresión verbal y el área de apoyo emocional; por último, hallaron correlación entre la subescala de sospecha y el área de reciprocidad.

\section{HIPÓTESIS}

\section{Hipótesis general}

El clima familiar y la agresividad de estudiantes de secundaria de Lima Metropolitana se relacionan positivamente.

\section{Hipótesis específicas}

$\mathrm{H}_{1}$. Existen diferencias significativas en el clima familiar de los estudiantes que cursan cuarto y quinto de secundaria en función del sexo.

$\mathrm{H}_{2}$. Existen diferencias significativas en la agresividad de los estudiantes que cursan cuarto y quinto de secundaria en función del sexo.

\section{MÉTODO}

En concordancia con la hipótesis formulada se empleó en un primer momento el método descriptivo con un diseño correlacional para relacionar las dos variables, y luego el diseño descriptivo comparativo para comparar los resultados de los alumnos en función del sexo.

\section{Sujetos}

Se tomó en forma estratificada y por cuotas una muestra de 237 estudiantes de ambos sexos, cuyas edades fluctuaron entre los 14 y 18 años, que cursaban cuarto y quinto de secundaria de centros educativos estatales de Lima Metropolitana. Las variables principales del estudio fueron: clima familiar y agresividad.

\section{Técnicas e instrumentos}

Dado el carácter de los fenómenos estudiados se utilizaron la técnica psicométrica y de observación indirecta.

Los instrumentos utilizados fueron:

1. Escala del clima social en la familia (FES), autores: R. H. Moos. y E. J. Trickett. La estandarización fue realizada por Cesar Ruiz Alva y Eva Guerra Turín (1993). La administración de la citada escala puede ser individual o colectiva, el tiempo de aplicación es en promedio 20 minutos. La significación es evaluar las características socioambientales y las relaciones personales en la familia. La tipificación se realiza 
mediante Baremos para la forma individual y grupo familiar, elaborados con muestras de Lima Metropolitana. Esta escala permite observar tres dimensiones: relaciones, desarrollo y estabilidad. La dimensión relaciones analiza las áreas cohesión, expresividad y conflictos. La dimensión desarrollo mide las áreas autonomía, actuación, intelectual-cultural y moralidad-religiosidad. La dimensión estabilidad se divide en organización y control.

Las modalidades de examen son: individual o colectiva, calificándose de acuerdo a la clave. Después, se usa el baremo individual (áreas y categorías) que sirve para el análisis final de los resultados.

Grupo familiar, para los padres e hijos adolescentes, se evalúa el grupo independientemente, marcando unos y otros sus respuestas. Luego se obtiene el puntaje promedio de cada una de las áreas y se califica vía la clave. Después se usa el baremo de grupo familiar (áreas y categorías) que servirá para el análisis final de los resultados.

Para la estandarización en Lima, usando el método de consistencia interna, los coeficientes de confiabilidad van de 0.80 a 0.91 con una media de 0.89 para el examen individual, siendo las áreas cohesión, intelectual-cultural, expresión y autonomía, las más altas. En el test-retest con 2 meses de lapso, los coeficientes eran en promedio 0.86 (variando de tres a seis puntos). La validez de la prueba se logró correlacionándola con la prueba de Bell, específicamente en el área de ajuste en el hogar (con adolescentes, los coeficientes fueron: en área cohesión 0.57 , conflicto 0.60 , organización 0.51 ) con adultos, los coeficientes fueron: cohesión 0.60 , conflicto 0.59 , organización 0.57 y expresión 0.53, en el análisis a nivel de grupo familiar. También se prueba el FES con la escala TAMAI (área familiar) y al nivel individual, los coeficientes fueron: en cohesión 0.62 , expresividad 0.53 y conflicto 0.59 .

2. Inventario de hostilidad de Buss-Durkee, propuesto por A. H. Buss en 1957, adaptado a nuestro país por Carlos Reyes R. en 1987. El test está constituido por un cuestionario que consta de 91 reactivos, distribuidos en 6 escalas. Cada subescala cuenta con 15 reactivos, excepto la subescala de irritabilidad que cuenta con 16 reactivos. La calificación permite obtener dos puntuaciones: una parcial, escala por escala o total. En las puntuaciones parciales por subtest oscila entre 0 a 15 (16 para irritabilidad) y en las puntuaciones totales de 15 a 91, la duración de la prueba es en promedio de 20 minutos y su objetivo primordial es evaluar el nivel de agresividad del sujeto. La estimación de la confiabilidad del cuestionario modificado de agresividad, se encontró a través del alpha de Cronbach, dando como resultado general 0.45. Para la estimación de la validez en términos de consistencia interna se utiliza el coeficiente producto momento de Pearson, y para precisar con exactitud la correlación se utilizó el corrector de McNemar, dando como resultado en la escala de irritabilidad 0.30; verbal 0.32 ; indirecta 0.31 ; física 0.27 ; resentimiento 0.32 ; sospecha 0.29 .

Técnicas de procesamiento de datos: se utilizaron las tablas de frecuencias para describir los resultados y medidas de tendencia central como la moda y la mediana. Para la contrastación de hipótesis se utilizó el Chi cuadrado para determinar la relación entre las variables clima familiar y agresividad, y la prueba de U de Mann-Withney para establecer diferencias en función del sexo y del grado de instrucción. 


\section{Procedimientos}

La recolección de la información se efectuó de manera colectiva, para el caso del clima social en la familia y la prueba de agresividad. Los instrumentos se administraron siguiendo las normas establecidas en los respectivos manuales.

Las respuestas fueron calificadas en forma manual y se hizo una base de datos que fue procesada mediante el paquete estadístico SPSS versión 13 en inglés.

\section{RESULTADOS}

\section{Resultados descriptivos}

Tabla N. ${ }^{\circ}$ 1. Resultados descriptivos de las dimensiones de la escala de clima social en la familia.

\begin{tabular}{lccccccccc}
\hline Categoría & & Relación & \multicolumn{3}{c}{ Desarrollo } & \multicolumn{3}{c}{ Estabilidad } \\
\hline & f & hi & Hi & f & hi & Hi & f & hi & Hi \\
\cline { 2 - 10 } Muy mala & 3 & 1.3 & 1.3 & 24 & 10.1 & 10.1 & 11 & 4.6 & 4,6 \\
Mala & 24 & 10.1 & 11.4 & 154 & 65.0 & 75.1 & 57 & 24.1 & 28,7 \\
Media & 180 & 75.9 & 87.3 & 54 & 22.8 & 97.9 & 163 & 68.8 & 97,5 \\
Buena & 30 & 12.7 & 100.0 & 5 & 2.1 & 100.0 & 6 & 2.5 & 100,0 \\
Muy buena & 0 & 0 & 100.0 & 0 & 0 & 100.0 & 0 & 0 & 100.0 \\
\hline Total & 237 & 100.0 & & 237 & 100.0 & & 237 & 100.0 & \\
\hline
\end{tabular}

En la Tabla N. ${ }^{\circ} 1$ se observan las frecuencias de los alumnos en las dimensiones de la escala de clima social familiar. La mayor frecuencia de los alumnos de la muestra (75\%) se ubica entre las categorías de mala y mala para el puntaje en la dimensión desarrollo. Respecto del puntaje en la dimensión relación, la muestra en un $75 \%$ se ubica entre la categoría media y buena. Es notorio que no se registre ninguna frecuencia para la categoría muy buena.

Tabla N. ${ }^{\circ}$ 2. Resultados descriptivos de los niveles en la dimensión relaciones de escala de clima social en la familia, según el sexo.

\begin{tabular}{lcccccc}
\hline & \multicolumn{3}{c}{ Mujeres } & \multicolumn{3}{c}{ Varones } \\
\hline \multirow{2}{*}{ Muy mala } & $\mathbf{f i}$ & $\mathbf{h i}$ & $\mathbf{H i}$ & $\mathbf{f i}$ & $\mathbf{h i}$ & $\mathbf{H I}$ \\
\cline { 2 - 7 } Mala & 1 & .8 & .8 & 2 & 1.8 & 1.8 \\
Media & 10 & 8.0 & 8.8 & 14 & 12.5 & 14.3 \\
Buena & 95 & 76.0 & 84.8 & 85 & 75.9 & 90.2 \\
Muy buena & 19 & 15.2 & 100.0 & 11 & 9.8 & 100.0 \\
\hline Total & 0 & 0 & & 0 & 0 & \\
\hline
\end{tabular}


Como se observa en la Tabla 2 tanto hombres, como mujeres se ubican con mayores porcentajes $(76 \%, 75.9 \%)$ en las categorías media y buena.

Tabla N. ${ }^{\circ}$ 3. Resultados descriptivos de los niveles en la dimensión desarrollo de escala de clima social en la familia, según el sexo.

\begin{tabular}{lcccccc}
\hline & \multicolumn{3}{c}{ Mujeres } & \multicolumn{3}{c}{ Varones } \\
\hline \multirow{2}{*}{ Muy mala } & $\mathbf{f i}$ & $\mathbf{h i}$ & $\mathbf{H I}$ & $\mathbf{f i}$ & $\mathbf{h i}$ & $\mathbf{H I}$ \\
\cline { 2 - 7 } Mala & 0 & 0 & 0.0 & 0 & 0 & 0.0 \\
Media & 8 & 6.4 & 6.4 & 16 & 14.3 & 14.3 \\
Buena & 88 & 70.4 & 76.8 & 66 & 58.9 & 73.2 \\
Muy buena & 27 & 21.6 & 98.4 & 27 & 24.1 & 97.3 \\
\hline Total & 2 & 1.6 & 100.0 & 3 & 2.7 & 100.0 \\
\hline & 125 & 100.0 & & 112 & 100.0 \\
\end{tabular}

En la Tabla 3 se observa que en la dimensión desarrollo tanto las mujeres, como los varones se ubican más frecuentemente entre las categorías media y buena, mostrándose un mayor porcentaje de varones (14.3\%) en la categoría mala, respecto de las mujeres $(6.4 \%)$.

Tabla N. ${ }^{0}$ 4. Resultados descriptivos de los niveles en la dimensión estabilidad de la escala de clima social en la familia, según el sexo.

\begin{tabular}{lcccccc}
\hline & \multicolumn{3}{c}{ Mujeres } & \multicolumn{3}{c}{ Varones } \\
\hline Muy mala & $\mathbf{f i}$ & $\mathbf{h i}$ & $\mathbf{H i}$ & $\mathbf{f i}$ & $\mathbf{h i}$ & $\mathbf{H i}$ \\
\cline { 2 - 7 } Mala & 6 & 4.8 & 4.8 & 5 & 4.5 & 4.5 \\
Media & 25 & 20.0 & 24.8 & 32 & 28.6 & 33,0 \\
Buena & 92 & 73.6 & 98.4 & 71 & 63.4 & 96.4 \\
Muy buena & 2 & 1.6 & 100.0 & 4 & 3.6 & 100.0 \\
\hline Total & 0 & 0 & & 0 & 0 & \\
\hline & 125 & 100.0 & & 112 & 100.0 \\
\end{tabular}

La Tabla 4 muestra resultados similares tanto para varones, como mujeres en la dimensión estabilidad al observarse que el $98.4 \%$ de la muestra de mujeres y al $96.4 \%$ de varones se ubican entre la categoría muy mala y media. 
Tabla N. ${ }^{0}$ 5. Resultados descriptivos del cuestionario de agresión de los estudiantes.

\begin{tabular}{lccccc}
\hline & AgrFis & Host & Ira & AgrVer & Total \\
\hline $\mathrm{N}$ & 237 & 237 & 237 & 237 & 237 \\
Media & 3.11 & 5.45 & 3.59 & 2.33 & 3.41 \\
Mediana & 3.22 & 5.40 & 3.57 & 2.38 & 3.38 \\
Moda & 3.22 & 5.20 & 3.71 & 2.50 & 3.21 \\
Desviación estándar & 0.540 & 0.418 & 0.285 & 0.294 & 0.278 \\
Varianza & 0.292 & 0.174 & 0.082 & 0.087 & 0.077 \\
\hline
\end{tabular}

En la Tabla 5 se aprecia que los alumnos de la muestra presentan en agresividad física media de 3.11, en hostilidad 5.45, en ira 3.59, en agresividad verbal 2.33, y en total 3.41. Además se observa que en agresividad física se presenta una mayor desviación de los datos con respecto a la media (0.540), una mayor variabilidad $(0.292)$ y el $50 \%$ presenta una puntuación mayor a 3.21. Los puntajes bajos encontrados en la subescala de agresividad verbal significan que no es una conducta que caracteriza a la muestra, no siendo el mismo caso para las escalas de agresividad física, hostilidad, ira y agresividad total.

Tabla N. ${ }^{\circ}$ 6. Resultados descriptivos de las subescalas del cuestionario de agresión, según el sexo.

\begin{tabular}{|c|c|c|c|c|c|c|c|c|c|c|}
\hline & \multicolumn{2}{|c|}{$\begin{array}{l}\text { Agresividad } \\
\text { física }\end{array}$} & \multicolumn{2}{|c|}{ Hostilidad } & \multicolumn{2}{|c|}{ Ira } & \multicolumn{2}{|c|}{$\begin{array}{c}\text { Agresividad } \\
\text { verbal }\end{array}$} & \multicolumn{2}{|c|}{ Total } \\
\hline & $\mathbf{M}^{*}$ & $\mathbf{V}^{* *}$ & $\mathbf{M}$ & $\mathbf{V}$ & $\mathbf{M}$ & $\mathbf{V}$ & $\mathbf{M}$ & $\mathbf{V}$ & $\mathbf{M}$ & $\mathbf{V}$ \\
\hline $\mathrm{N}$ & 125 & 112 & 125 & 112 & 125 & 112 & 125 & 112 & 125 & 112 \\
\hline Media & 3.09 & 3.11 & 5.45 & 5.45 & 3.58 & 3.69 & 2.33 & 2.33 & 3.41 & 3.62 \\
\hline Mediana & 3.22 & 3.22 & 5.40 & 5.40 & 3.57 & 3.64 & 2.38 & 2.44 & 3.38 & 3.46 \\
\hline Moda & 3.22 & 3.22 & 5.20 & 5.20 & 3.71 & 3,71 & 2.50 & 2.50 & 3.21 & 3.34 \\
\hline Desviación estándar & .545 & .536 & .418 & .418 & .287 & .283 & .295 & .294 & .279 & .278 \\
\hline Varianza & .298 & .288 & .175 & .175 & .083 & .081 & .087 & .087 & .078 & .077 \\
\hline
\end{tabular}

${ }^{*}$ mujeres

${ }^{* *}$ varones

En la Tabla 6 se observan las medidas de tendencia central de la agresividad en función del sexo, observándose que los puntajes más altos corresponden para la subescala de hostilidad (5.45) tanto para varones como para mujeres. Los puntajes bajos se muestran en la subescala de agresividad verbal lo que significa que no es una conducta que caracteriza a la muestra, no siendo el mismo caso para las escalas agresividad física, hostilidad, ira y agresividad total. 


\section{Resultados inferenciales}

Tabla N. ${ }^{0}$ 7. Prueba de Kolmogorov-Smirnov de bondad de ajuste a la curva normal para los resultados de las subescalas de agresividad y las dimensiones de clima social en la familia.

\begin{tabular}{lcccccccc}
\hline & \multicolumn{3}{c}{ Agresividad } & \multicolumn{5}{c}{ Clima familiar } \\
\hline & AgrFis & Host & Ira & AgrVer & Relac & Desar & Estab & $\begin{array}{l}\text { Clima } \\
\text { social }\end{array}$ \\
\cline { 2 - 9 } & Kolmogorov- \\
$\begin{array}{l}\text { Smirnov Z } \\
\mathrm{p}\end{array}$ & 3.731 & 2.346 & 2.424 & 3.896 & 3.570 & 2.590 & 1.710 & 3.111 \\
& .000 & .000 & .000 & .000 & .000 & .000 & .006 & .000 \\
\hline
\end{tabular}

Se observa de la Tabla 7, un nivel crítico (significación asintótica bilateral $=\mathrm{p}$ ) menor a 0.05 en los subtests que constituyen la agresividad y el clima social en la familia, por lo que se rechaza la hipótesis de normalidad y se concluye que las puntuaciones de estos subtests no se ajustan a una distribución normal.

Tabla N. ${ }^{\circ}$ 8. Relación entre los resultados de la escala de clima social familiar y las subescalas del cuestionario de agresión.

\begin{tabular}{|c|c|c|c|c|c|c|c|c|c|}
\hline & \multicolumn{3}{|c|}{ Agresividad física } & \multicolumn{2}{|c|}{ Hostilidad } & \multicolumn{2}{|c|}{ Ira } & \multicolumn{2}{|c|}{ Agresividad verbal } \\
\hline & gl & Valor & $\mathbf{p}$ & Valor & $\mathbf{p}$ & Valor & $\mathbf{p}$ & Valor & $\mathbf{p}$ \\
\hline Chi-cuadrado de Pearson & 6 & 5.004 & .543 & 1.426 & .700 & 5.725 & .513 & 13.243 & $.041 *$ \\
\hline Razón de verosimilitud & 6 & 5.020 & .541 & 1.259 & .739 & 5.712 & .511 & 12.327 & .057 \\
\hline N. ${ }^{\circ}$ de casos válidos & & 237 & & 237 & & 237 & & 237 & \\
\hline
\end{tabular}

Se observa en la Tabla 8, el estadístico Chi cuadrado de Pearson toma un valor de: 5.004 en el caso de agresividad física, con una probabilidad asociada $(\mathrm{p}=$ significación asintótica) de 0.543; en el caso de hostilidad es 1.426, con una probabilidad asociada de 0.700 ; en el caso de ira es 5.725 , con una probabilidad asociada de 0.513 , y en el caso de agresividad verbal es 7.243, con una probabilidad asociada de 0.065 . Puesto que la probabilidad es menor a 0.05 en el caso de agresividad verbal, decidimos rechazar la hipótesis de independencia y concluir que las variables clima social familiar y agresividad verbal están relacionados. 
Tabla $\mathbf{N}^{0}$ 9. Relación entre la dimensión relaciones de la escala clima social en la familia y las subescalas del cuestionario de agresión.

\begin{tabular}{|c|c|c|c|c|c|c|c|c|c|}
\hline & \multicolumn{3}{|c|}{ Agresividad física } & \multicolumn{2}{|c|}{ Hostilidad } & \multicolumn{2}{|c|}{ Ira } & \multicolumn{2}{|c|}{ Agresividad verbal } \\
\hline & gl & Valor & $\mathbf{p}$ & Valor & $\mathbf{p}$ & Valor & $\mathbf{p}$ & Valor & $\mathbf{p}$ \\
\hline Chi-cuadrado de Pearson & 6 & 1,319 & ,971 & 13,664 &, $048 *$ & 7,682 &, 096 & 13,179 &, $040 *$ \\
\hline Razón de verosimilitud & 6 & 1,558 & ,956 & 13,350 &, 049 & 6,745 &, 098 & 15,575 &, 016 \\
\hline $\mathrm{N}^{\circ}$ de casos validos & & 237 & & 237 & & 237 & & 237 & \\
\hline
\end{tabular}

Vemos que en la Tabla $\mathrm{N}^{\circ}$ 9, el estadístico chi-cuadrado de Pearson toma un valor de: 1,319 en el caso de Agresividad Física, con una probabilidad asociada ( $\mathrm{p}=$ Significación asintótica) de 0,971; en el caso de Hostilidad es 3,664, con una probabilidad asociada de 0,030; en el caso de Ira es 4,329, con una probabilidad asociada de 0,049; y en el caso de Agresividad verbal es 13,179, con una probabilidad asociada de 0,040. Puesto que la probabilidad es menor a 0,05 en los casos de Hostilidad e Ira, decidimos rechazar la hipótesis de independencia y concluir que las variables Relación y Hostilidad; relación y Agresividad Verbal están relacionadas.

Tabla N¹0. Relación entre la dimensión Desarrollo de la Escala Clima Social en la Familia y las subescalas del cuestionario de agresión.

\begin{tabular}{|c|c|c|c|c|c|c|c|c|c|}
\hline & \multicolumn{3}{|c|}{ Agresividad física } & \multicolumn{2}{|c|}{ Hostilidad } & \multicolumn{2}{|c|}{ Ira } & \multicolumn{2}{|c|}{ Agresividad verbal } \\
\hline & gl & Valor & $\mathbf{p}$ & Valor & $\mathbf{p}$ & Valor & $\mathbf{p}$ & Valor & $\mathbf{p}$ \\
\hline Chi-cuadrado de Pearson & 6 & 6.502 & .090 & 1.279 & .734 & 1.379 & .755 & 7.182 & .066 \\
\hline Razón de verosimilitud & 6 & 6.527 & .089 & 1.150 & .765 & 1.350 & .733 & 6.745 & .080 \\
\hline N. ${ }^{\circ}$ de casos válidos & & 237 & & 237 & & 237 & & 237 & \\
\hline
\end{tabular}

Se observa en la Tabla 10, el estadístico Chi cuadrado de Pearson toma un valor de: 6.502 en el caso de agresividad física, con una probabilidad asociada ( $\mathrm{p}=$ significación asintótica) de 0.090; en el caso de hostilidad es 1.279, con una probabilidad asociada de 0.734; en el caso de ira es 1.379 , con una probabilidad asociada de 0.755 , y en el caso de agresividad verbal es 7.182 , con una probabilidad asociada de 0.066 . Puesto que la probabilidad es mayor a 0.05 en todos los casos, decidimos aceptar la hipótesis de independencia y concluir que las variables desarrollo y las subescalas de agresión no están relacionadas. 
Tabla $\mathbf{N}^{\circ}$ 11. Relación entre la dimensión estabilidad de la escala clima social en la familia y las subescalas del cuestionario de agresión.

\begin{tabular}{|c|c|c|c|c|c|c|c|c|c|}
\hline & \multicolumn{3}{|c|}{ Agresividad física } & \multicolumn{2}{|c|}{ Hostilidad } & \multicolumn{2}{|c|}{ Ira } & \multicolumn{2}{|c|}{ Agresividad verbal } \\
\hline & gl & Valor & $\mathbf{p}$ & Valor & $\mathbf{p}$ & Valor & $\mathbf{p}$ & Valor & $\mathbf{p}$ \\
\hline Chi-cuadrado de Pearson & 6 & 3.098 & .796 & 1.198 & .753 & 1.219 & .783 & 1.663 & .645 \\
\hline Razón de verosimilitud & 6 & 4.099 & .663 & 1.029 & .794 & 1.202 & .769 & 1.775 & .620 \\
\hline N. ${ }^{\circ}$ de casos válidos & & 237 & & 237 & & 237 & & 237 & \\
\hline
\end{tabular}

Vemos que en la Tabla 11, el estadístico Chi-cuadrado de Pearson toma un valor de: 3.098 en el caso de agresividad física, con una probabilidad asociada ( $\mathrm{p}=$ significación asintótica) de 0.796; en el caso de hostilidad es 1.198, con una probabilidad asociada de 0.753; en el caso de ira es 1.219 , con una probabilidad asociada de 0.783 , y en el caso de agresividad verbal es 1.663 , con una probabilidad asociada de 0.620 . Puesto que la probabilidad es mayor a 0.05 en todos los casos, decidimos aceptar la hipótesis de independencia y concluir que las variables estabilidad y las subescalas de agresión no están relacionadas.

Tabla N. ${ }^{\circ}$ 12. Diferencia de medias (U Mann Whitney) de las dimensiones de clima social en la familia en los estudiantes, según el sexo.

\begin{tabular}{lcccc}
\hline & Relac. & Desar. & Estab. & Total \\
\cline { 2 - 5 } Mann-Whitney U & 6414.000 & 6834.000 & 5804.000 & 6451.000 \\
Z & -1.156 & -.321 & -2.285 & -1.079 \\
Asymp. Sig. (2-tailed) & .248 & .748 & $.022 *$ & .281 \\
\hline
\end{tabular}

En la Tabla 12 se analiza el estadístico U de Mann-Whitney, en las áreas que conforman clima social familiar, el subtest de estabilidad de la tipificación de hombres y mujeres vale $\mathrm{Z}=-2.285$ y $\mathrm{p}=0.022$. Debido a que el nivel crítico bilateral (p) es menor a 0.05 en el caso de estabilidad, podemos rechazar la hipótesis de igualdad de promedios, y concluir que hombres y mujeres presentan diferencias significativas en la dimensión estabilidad del clima social familiar.

Tabla N. ${ }^{0}$ 13. Diferencia de medias (U Mann Whitney) de las subescalas del cuestionario de agresividad en los estudiantes, según el sexo.

\begin{tabular}{lcccc}
\hline & Agr. Fís. & Host. & Ira & Agr. Ver. \\
\cline { 2 - 5 } Mann-Whitney U & 6979.000 & 6844.500 & 6075.000 & 6309.000 \\
$\mathrm{Z}$ & -.968 & -.298 & -1.801 & -1.361 \\
$\mathrm{p}$ & $.041^{*}$ & .765 & .072 & .174 \\
\hline
\end{tabular}


La Tabla 13 ofrece el estadístico U de Mann-Whitney, en las áreas que conforman el cuestionario de agresividad. En el caso de agresividad física, la tipificación de ambos vale $\mathrm{Z}=-0.968$ y $\mathrm{p}=0.041$. Observándose que entre los componentes de agresión, el nivel crítico bilateral (p) es menor a 0.05 en el caso de agresividad física; por tanto, podemos rechazar la hipótesis de igualdad de promedios, y concluir que hombres y mujeres presentan diferencias significativas en la subescala de agresividad física.

\section{ANÁLISIS Y DISCUSIÓN}

Al procesar los datos obtenidos en la escala de clima social familiar se encuentra el siguiente panorama: la mayor frecuencia (85.2\%) de alumnos de la muestra perciben el clima familiar en la dimensión desarrollo entre muy mala y mala (véase Tabla 1); es decir, los alumnos perciben que en su familia no hay condiciones adecuadas para desarrollar la autonomía, la seguridad de sí mismos, ni oportunidades para la participación en actividades recreativas, ni incentivos para el desarrollo intelectual y político. Estos resultados se condicen con los de Zavala (2001), quien halló que el $47.6 \%$ de los evaluados comunica valores que los ubica entre muy mala y mala señalando que el tipo de relaciones que se establecen al interior de la familia no permiten la formación o el aprendizaje de intereses hacia el estudio y el desarrollo personal.

Del mismo modo, se encontró un considerable porcentaje $(88.6 \%)$ de alumnos que se ubican entre las categoría media y buena para la dimensión relación. Lo que revela que los alumnos consideran aceptable la comunicación y la libre expresión de los sentimientos dentro de la familia, los alumnos perciben un adecuado nivel de cohesión que les permite compenetrarse con los demás miembros de su familia. Es notorio que no se registre ninguna frecuencia para la categoría muy buena en los diversos componentes de la escala de clima social familiar (véase Tabla 1). Estos resultados difieren de los de Zavala (2001), quien en la misma dimensión encontró que el 39.2\% de la muestra evaluada perciben las relaciones entre la categoría mala y muy mala.

Cuando se examinan los resultados descriptivos de la escala de clima social familiar en función del sexo, se observa que en la dimensión desarrollo se registra un mayor porcentaje de varones que categorizan como malo el desarrollo personal dentro de sus familias; contrariamente, las mujeres en su mayoría perciben como media a buena las posibilidades de desarrollo dentro de su familia (véase Tabla 3). Resultados similares se registran en la dimensión estabilidad, las mujeres en un mayor porcentaje $(74.6 \%)$ que los varones $(69 \%)$ perciben que el grado de organización y control en sus familias es categorizado como medio a bueno, en contraposición a los varones quienes en mayor porcentaje que las mujeres lo categorizan como malo y muy malo (véase Tabla 4).

Al analizar los resultados del puntaje general de la escala de clima social familiar con las subescalas del cuestionario de agresividad, se encuentra que existe relación entre el clima social familiar y la subescala agresividad verbal (véase Tabla 8). En la presente investigación se ha evaluado el clima familiar tal como lo perciben los adolescentes, esta variable y el hecho de que los alumnos tienden a mostrar agresividad verbal están correlacionados. 
Al correlacionar cada una de las dimensiones de la escala de clima social familiar con las subescalas de agresividad, se encontró relación entre la dimensión relaciones de la escala clima social en la familia con la subescala de hostilidad del cuestionario de agresividad (véase tabla 9). Esto significa que el grado de comunicación y libre expresión dentro de la familia y el grado de interacción conflictiva que la caracteriza se relaciona con el factor conductual manifestado en agresividad verbal y el factor cognitivo de la agresividad evidenciado en la hostilidad (Buss y Perry, 1992). Las dimensiones desarrollo y estabilidad de la escala clima social en la familia no revelaron relaciones significativas con ninguna de las subescalas del cuestionario de agresividad (véase tablas 10 y 11).

Cuando se realiza la comparación de la dimensión estabilidad entre los varones y mujeres de la muestra se hallaron diferencias estadísticamente significativas (véase Tabla 4 y Tabla 12). Lo que indica que en este estudio, las mujeres otorgan mayor importancia a una clara organización y estructura, participan más activamente al planificar las actividades y responsabilidades de la familia, consideran además que la dirección de la vida familiar se atiene a reglas y procedimientos establecidos (Ruiz y Guerra, 1993).

Analizando la agresividad de hombres y mujeres se han encontrado diferencias significativas en la subescala de agresividad física. Estos resultados se condicen con los resultados de Díaz (1987), Hernández (1988), Castrillón y Vieco (2002), Kaleel, Justicia, Benítez y Pichardo (2007). En todas las investigaciones antes mencionadas los hombres mostraron más agresividad física que las mujeres. Posiblemente la explicación a este hallazgo se encuentre en el hecho de que desde la perspectiva biológica, el genotipo de una persona y otros correlatos biológicos del género pueden influir en la propensión a la conducta agresiva, antisocial (Castillo, 2006). Sin embargo, para Shaffer (2000), citado por Espinosa (2009), la familia cumple un rol preponderante en la determinación de las conductas distintivas de cada género. Desde la primera infancia presentan esterotipos muy marcados para cada uno, siendo el del varón: dominante, agresivo, audaz, y la niña cariñosa, sensible suave.

En síntesis, los resultados de la investigación muestran que el clima social familiar se correlaciona con la agresividad y esto se condice con hallazgos de Díaz (1987) y Hernández (1988), quienes encontraron que las actitudes parentales y la conducta agresiva se hallan correlacionadas. En este mismo sentido, Noroño, Cruz, Cadalso y Fernández (2002) encontraron que las características del medio familiar influyen en las características agresivas de los niños. Los teóricos de la agresividad concuerdan con estos hallazgos; en este sentido, Gerard Patterson, citado por Castillo (2006), afirma que el entorno familiar es la esfera principal en el aprendizaje del comportamiento agresivo, por ser el más cercano al niño y el que mayor influencia produce en él (Espinosa, 2009). Por su parte, Buss, A. y Perry (1992) afirman que "cuando en la familia se intentan solucionar los problemas con agresividad y enfrentar la agresividad con agresividad", los niños fácilmente relacionan la fuerza con la consecución del objetivo y ven que la fuerza funciona de una forma muy efectiva para convencer y controlar a otros. Gaspar (1999) explica que la socialización de la agresividad tiene que ocurrir en el ámbito familiar si hay fallas en ese entorno o el ambiente familiar natural. 


\section{CONCLUSIONES}

1. Se ha encontrado relación entre el clima social en la familia y la agresividad de estudiantes de secundaria.

2. Existe relación entre la dimensión relaciones de la escala de clima social en la familia con las subescalas hostilidad y agresividad verbal del cuestionario de agresividad.

4. No se halló relación significativa entre la dimensión desarrollo de la escala de clima social en la familia y las subescalas del cuestionario de agresividad.

5. No se halló relación significativa entre la dimensión estabilidad del clima social en la familia y las subescalas del cuestionario de agresividad.

6. El clima social familiar de los estudiantes se muestra diferente en función del sexo al hallarse diferencias significativas en la dimensión estabilidad entre varones y mujeres.

7. La agresividad de los estudiantes se muestra diferente en función del sexo al hallarse diferencias significativas en la subescala de agresividad física entre varones y mujeres.

\section{REFERENCIAS BIBLIOGRÁFICAS}

1. Benites, L. (1997). Tipos de familia, habilidades sociales y autoestima en un grupo de adolescentes en situación de riesgo. En Revista Cultura UPSMP, 12.

2. Berkowitz (1996). Agresión. Causas, consecuencias y control. Bilbao: Descleé de Briuwer.

3. Buss, A. H. y Perry, M. (1992). Cuestionario de agresión. En Journal of personality and social psychology, 63, 452-459.

4. Castillo, M. (2006). El comportamiento agresivo y sus diferentes enfoques. En Revista de la Universidad Simon Bolivar, 9, 15, 66-170.

5. Castrillón, M. y Vieco, D. (2002). Actitudes justificativas del comportamiento agresivo y violento en estudiantes universitarios de la ciudad de Medellín, Colombia. En Revista de la Facultad de Salud Pública, 20, 2, 51-66.

6. Díaz, L. (1987). Diagnóstico situacional del manejo de contingencias familiares: un estudio exploratorio en un grupo de familias de Lima Metropolitana, (tesis de maestría en ciencias con mención en psicología). Lima: UPCH.

7. Escriva, V.; García, P. y Pérez, E. (2001). Clima familiar y desarrollo del autoconcepto: un estudio longitudinal en población adolescente. En Revista Latinoamericana de Psicología, 33, 3, 243-259.

8. Espinosa, M. A. (2009). Roles de género y modelos familiares. Recuperado en http:// www.sare-emakunde.com/media/anual/archivosAsociados/Espinosa.M_05_es.pdf, el día 18.04.10, 14:29 p.m. 
9. García, C. (2005). Habilidades sociales, clima social familiar y rendimiento académico en estudiantes universitarios. En Revista de la Facultad de Psicología de la Universidad San Martín de Porres, LIBERABIT, 11, 63-74.

10. Gaspar (1999). Orígenes y evolución de la agresividad y de la violencia en la adolescencia. En Boletín del Instituto interamericano del niño. Infancia, 70, 237, 21-30.

11. Guerra, E. (1993). Clima social familiar en adolescentes y su influencia en el rendimiento académico, (tesis). Lima: UNMSM.

12. Hernández, R. (1988). Influencia del ambiente familiar y de las actitudes parentales en las conductas agresivas del preescolar: estudio realizado en el Instituto de salud mental "Honorio Delgado-Hideyo Noguchi, (tesis de bachillerato en enfermería). Lima: UPCH.

13. Hurlock, E. (2000). Psicología de la adolescencia. Buenos Aires: Paidós.

14. Jugo, M. y Chávez, G. (2004). Relación entre el clima social familiar y niveles de agresividad en los alumnos de quinto año de secundaria en el centro educativo estatal mixto Gustavo Ries de la ciudad de Trujillo, (tesis de licenciatura en psicología). Lima: Universidad César Vallejo.

15. Jimenez, M.; Ferró, M.; Gómez, R. y Parra, P. (1999). Evaluación del clima familiar en una muestra de adolescentes. En Revista de psicología general y aplicada de la Universidad de Murcia, 52, 4, 453-462.

16. Kaleel, A.; Justicia, F.; Benítez, J. y Pichardo, M. (2007). Incidencia de los malos tratos entre iguales en alumnos de Hebrón (Palestina). En Revista del colegio de psicología de Andalucía Occidental y la Universidad de Sevilla. Apuntes en psicología, $25,2,175-184$.

17. Lazarus, R. y Lazarus, B. (2000). Pasión y razón: la comprensión de nuestras emociones. Barcelona: Paidós.

18. Montiel-Nava, C.; Montiel-Barbero, I. y Peña, J. (2005). El efecto del clima familiar en el desarrollo del trastorno por déficit de atención-hiperactividad (TDAH). En Revista de psicología conductual, 13, 2, 297-310.

19. Moreno, M., Vacas, C. y Roa, J. (2006). Victimización escolar y clima socio-familiar. En Revista iberoamericana de educación OEI, 41, 1.

20. Murcia, H.; Reyes, A.; Gómez, L.; Medina, F.; Paz, B. y Fonseca, P. (2007). Caracterización familiar de escolares que presentan comportamiento hostil-agresivo en escuelas públicas y privadas del distrito central. En Revista de la facultad de ciencias médicas de la Universidad Autónoma de Honduras. Suplemento N. ${ }^{o} 1$.

21. Muñoz, J.; Carreras, M. y Braza P. (2004). Aproximación al estudio de las actitudes y estrategias de pensamiento social y su relación con los comportamientos disruptivos en el aula en la educación secundaria. Revista anales de psicología de la Universidad de Cadiz, 20, 1, 81-91. 